\title{
Applied Cryptography and Noise Resistant Data Security
}

\author{
Iqtadar Hussain $\mathbb{D}^{1}$, Fawad Ahmed, ${ }^{2}$ Umar M. Khokhar, ${ }^{3}$ and Amir Anees $\mathbb{D}{ }^{4}$ \\ ${ }^{1}$ Department of Mathematics, Statistics and Physics, College of Arts and Sciences, Qatar University, Doha, Qatar \\ ${ }^{2}$ Department of Electrical Engineering, HITEC University, Taxila, Pakistan \\ ${ }^{3}$ University System of Georgia, Georgia, USA \\ ${ }^{4}$ Department of Computer Science, La Trobe University, Melbourne, Australia
}

Correspondence should be addressed to Iqtadar Hussain; iqtadarqau@qu.edu.qa

Received 8 November 2018; Accepted 8 November 2018; Published 2 December 2018

Copyright (C) 2018 Iqtadar Hussain et al. This is an open access article distributed under the Creative Commons Attribution License, which permits unrestricted use, distribution, and reproduction in any medium, provided the original work is properly cited.

There have been tremendous efforts exerted in field of secure communication by mathematicians and computer scientists. However, at the same time, there are security concerns that still must be properly addressed. Moreover, a critical requirement for a cipher is to be distortion tolerant. One of the most sophisticated ciphers, the Advanced Encrypted Standard (AES), is still unbreakable and widely used in all kinds of industrial applications. However, as the case with other modern and secured ciphers, the AES cannot tolerate any sort of channel noise. The solution for noise corruption is usage of error detection and correction modules, but it will significantly increase the overall computational complexity and may not be required in low profile applications. The other efficient solution can be the Noise Resistant Ciphers which should have the inherit property of tolerating any sort of noise. Besides for security protocols to be secure, robust, and noise resistant, power constraints should also be considered. In this regard, lightweight ciphers are of great importance to be synchronized with the modern hardware platforms. However, due importance to security attacks is also necessary in designing the lightweight ciphers. The goal followed in this special issue is to create a volume of recent works on advances in all aspects of cryptography, especially towards those ciphers which can tolerate channel noise. Besides data encryption, the focus is also on data hiding and copyright protection techniques as well. We have selected eleven research articles which deal with different aspects of cryptography:
In the paper entitled "High Embedding Capacity Data Hiding Algorithm for H.264/AVC Video Sequences without Intraframe Distortion Drift," D.-C. Nguyen et al. proposed a high-quality data hiding algorithm based on H.246/AVC without intraframe distortion drift in which quantized coefficients are clustered into two different groups.

In the paper entitled "A Robust Watermarking Scheme for Online Multimedia Copyright Protection Using New Chaotic Map," A. Anees et al. worked on copyright protection for contents of digital video. The proposed scheme can be applied on the other kinds of data as well, such as text, audio, and digital images.

In the paper entitled "A Vendor-Neutral Unified Core for Cryptographic Operations in $\mathrm{GF}(\mathrm{p})$ and $\mathrm{GF}\left(2^{\mathrm{m}}\right)$ Based on Montgomery Arithmetic," M. Schramm et al. proposed a comprehensive adaptable hardware structure for efficient prime finite field and binary finite field arithmetic operations that expand the capabilities of single Montgomery Multiplier hardware designs.

In the paper entitled "A Novel Multiple-Bits Collision Attack Based on Double Detection with Error-Tolerant Mechanism," Y. Yuan et al. proposed a multiple-bits sidechannel collision attack based on double distance voting detection and an improved version, involving the errortolerant mechanism, which can find all 120 relations among 16 key bytes when applied to the AES.

In the paper entitled "An Efficient Certificateless Generalized Signcryption Scheme," B. Zhang et al. presented an 
efficient certificateless generic signcryption scheme without utilizing bilinear pairing operations.

In the paper entitled "LWR-Based Fully Homomorphic Encryption, Revisited," F. Luo et al. presented the first workable LWR-based FHE scheme. Their FHE scheme erases the expensive Gaussian noise sampling and thus be an alternative to the LWE-based FHEs.

In the paper entitled "To Study the Effect of the Generating Polynomial on the Quality of Nonlinear Components in Block Ciphers," S. Mahmood et al. proposed a new design of S-box which has good properties such as nonlinearity, strict avalanche, bit independence, linear approximation probability, and differential approximation probability.

In the paper entitled "Side-Channel Attacks and Countermeasures for Identity-Based Cryptographic Algorithm SM9," Q. Zhang et al. discussed the implementation of SM9 algorithm and its Simple Power Attack and then presented the template attack and fault attack on SPA-resistant SM9.

In the paper entitled "Analysis of Software Implemented Low Entropy Masking Schemes," D. Li et al. analyzed the vulnerabilities on the mask sets of software Low Entropy Masking Schemes implementations.

In the paper entitled "Understanding Keystroke Dynamics for Smartphone Users Authentication and Keystroke Dynamics on Smartphones Built-In Motion Sensors," H. Lee et al. evaluated features with motion data and without motion data using various features including keystroke data such as time interval and motion data.

In the paper entitled "Under Quantum Computer Attack: Is Rainbow a Replacement of RSA and Elliptic Curves on Hardware?," H. Yi presented techniques to exploit Rainbow signature on hardware meeting the requirements of efficient high-performance applications.

\section{Conflicts of Interest}

The authors declare that they have no conflicts of interest.

\section{Acknowledgments}

We would also like to thank all the reviewers who have participated in the review process of the articles submitted to this special issue and the special issue coordinators and acknowledge the technical support from the publishing team.

Iqtadar Hussain

Fawad Ahmed

Umar M. Khokhar

Amir Anees 


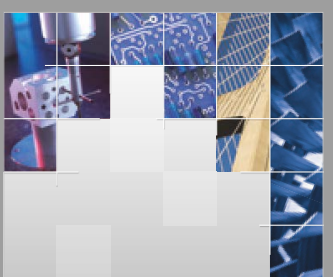

\section{Enfincering}
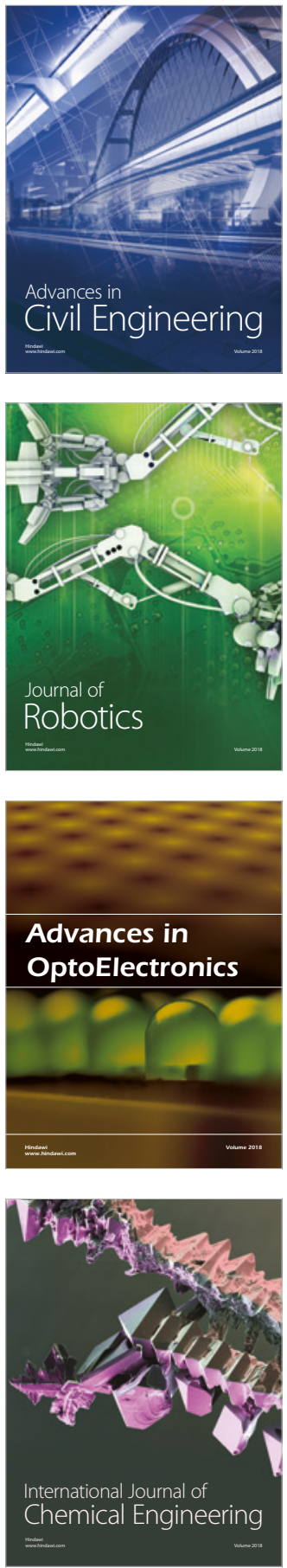

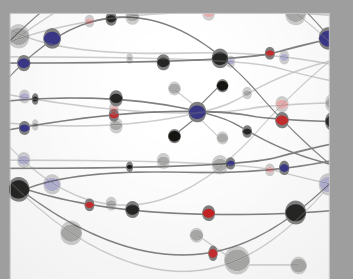

\section{Rotating \\ Machinery}

The Scientific World Journal

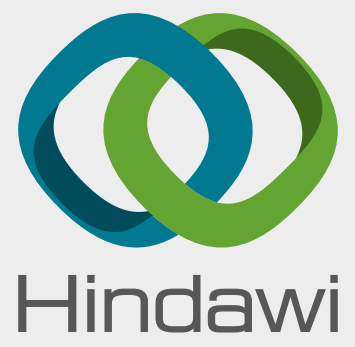

Submit your manuscripts at

www.hindawi.com
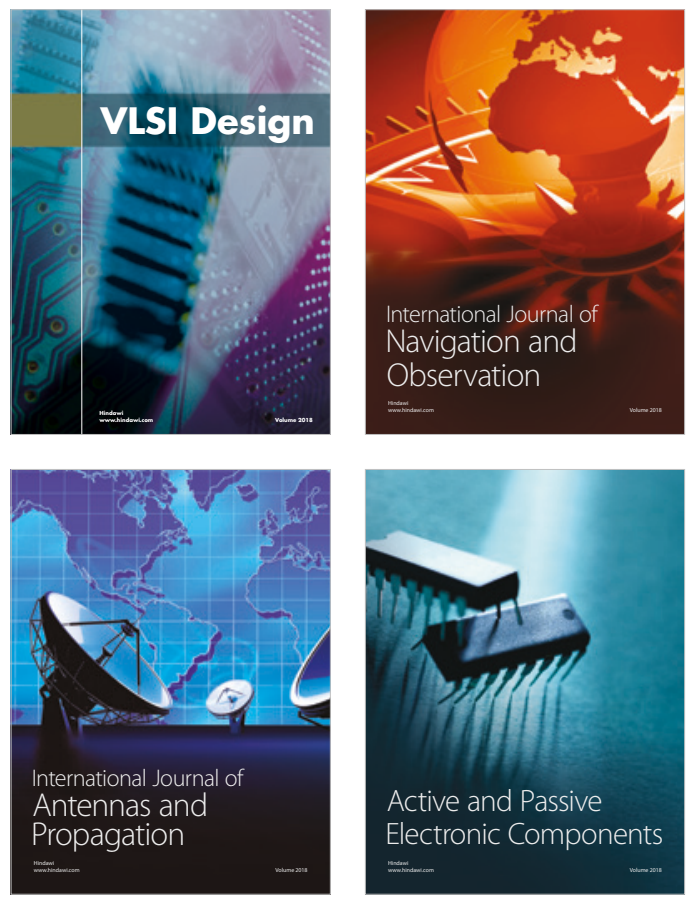
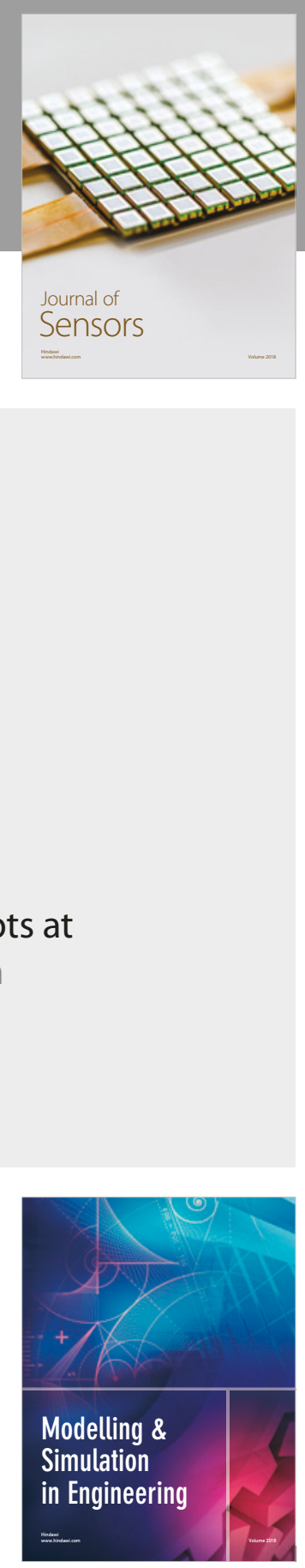

\section{Advances \\ Multimedia}
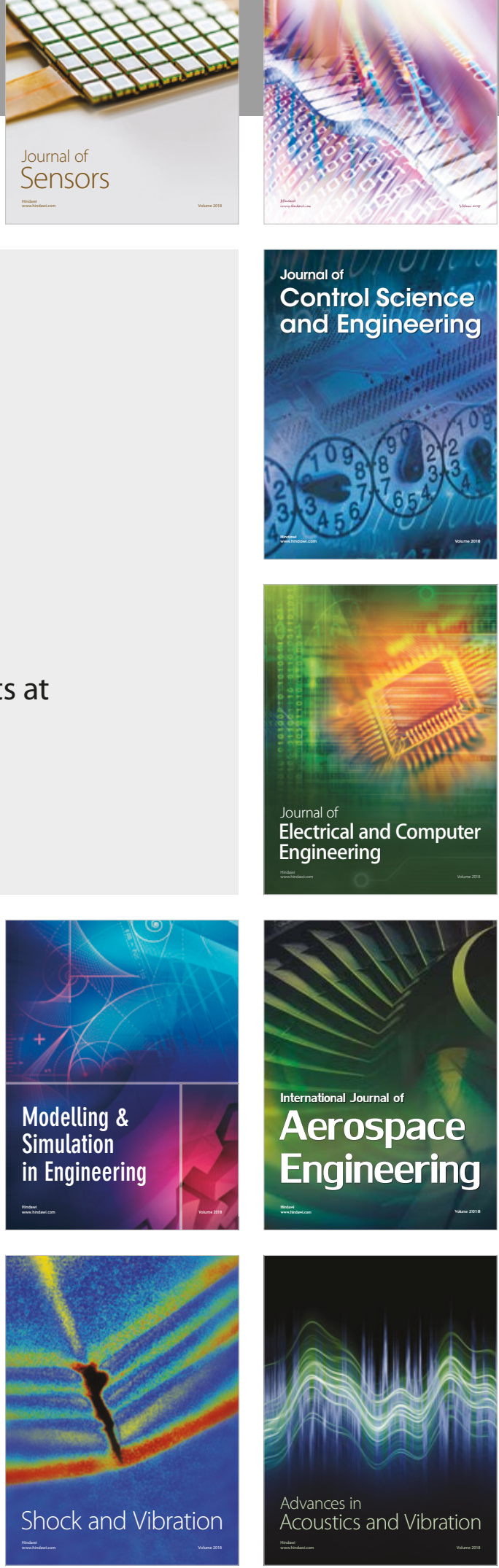